\title{
Docencia y comprensión situacional. El caso de una facultad de educación en el sureste de México
}

\author{
Teaching and situational understanding. The case of a Faculty of \\ Education in the southeast of Mexico
}

\section{Ensino e compreensão situacional. $O$ caso de uma faculdade de educação no sudeste do México}

\author{
Galo Emanuel López Gamboa \\ Universidad Autónoma de Yucatán, México \\ galo.lopez@correo.uady.mx \\ https://orcid.org/0000-0001-5581-7489 \\ Silvia Alejandra Baeza Aldana \\ Universidad Autónoma de Yucatán, México \\ alejandra.baeza@correo.uady.mx \\ https://orcid.org/0000-0001-6889-5355
}

\section{Resumen}

Este artículo presenta los resultados de un estudio realizado con motivo de la suspensión de labores docentes y académicas en una facultad de educación del sureste de México. Para ello, se parte de la idea de que los docentes cuya formación se basa en la pedagogía y en las ciencias de la educación conciben el fenómeno educativo de forma diferente y, en consecuencia, son capaces de anticipar, adecuar y tomar decisiones pertinentes que van más allá del traslado del aula presencial a la virtualidad con las respectivas herramientas tecnológicas; es decir, tienen no solo una consciencia de la situación, sino también una comprensión situacional que los conduce a la modificación de contenidos, de estrategias de enseñanza-aprendizaje y — de ser necesario — de criterios de evaluación, dado que la situación es extraordinaria y la enseñanza debe tomar en cuenta las posibilidades, pero también las restricciones que no pueden ser ignoradas. En ese sentido, en cuanto a la consciencia situacional, se encontró que todos los docentes lograron establecer satisfactoriamente la comunicación con sus estudiantes a través de diferentes 
Revista Iberoamericana de las Ciencias Sociales y Humanísticas

vías y redes, tanto institucionales como personales. Asimismo, en términos de la comprensión situacional, el profesorado señaló cambios importantes a partir de los efectos de la pandemia en la vida estudiantil, familiar y personal del alumnado, y se mostró sensible a la situación al grado de confrontar ellos mismos su rol docente y hacer los cambios necesarios para adaptarse lo mejor posible a una situación por demás retadora en múltiples niveles.

Palabras clave: comprensión situacional, conciencia situacional, educación superior, emergencia sanitaria.

\begin{abstract}
This article presents the results of a study carried out on the occasion of the suspension of teaching and academic work in a Faculty of Education in the southeast of Mexico. It starts from the premises that teachers whose basic training is pedagogy and educational sciences, conceive the educational phenomenon in a different way and, consequently, are capable of anticipating, adapting and making relevant decisions that go beyond the transfer of the virtual classroom with the respective technological tools; In other words, it does imply an awareness of the situation, but beyond that, a situational understanding that leads to the modification of content, teaching and learning strategies and even - if necessary - evaluation criteria, since the situation is extraordinary and emergency remote teaching has many possibilities but also restrictions that cannot be ignored. In this sense, in terms of situational awareness, it was found that all teachers were able to satisfactorily establish communication with their students through different channels and networks, both institutional and personal; Likewise, in terms of situational understanding, the teaching staff pointed out important changes from the effects of the pandemic on the student, family and personal life of the students and was sensitive to the situation to the extent of confronting their teaching role themselves and making the changes needed to best adapt to an otherwise challenging situation on multiple levels.
\end{abstract}

Keywords: situational understanding, situational awareness, higher education, health emergency. 
Revista Iberoamericana

de las Ciencias Sociales y

Humanísticas

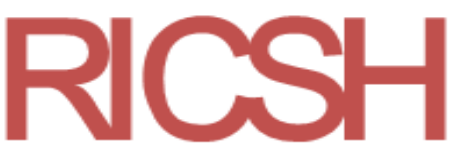

ISSN: $2395-7972$

\section{Resumo}

Este artigo apresenta os resultados de um estudo realizado por ocasião da suspensão do trabalho docente e acadêmico em uma faculdade de educação do sudeste do México. Para isso, parte-se pensa que os professores cuja formação se baseou na pedagogia e nas ciências da educação concebem o fenômeno educacional de forma diferenciada e, conseqüentemente, são capazes de se antecipar, se adaptar e tomar decisões pertinentes que também irão transferir a sala de aula para a virtualidade com as respectivas ferramentas tecnológicas; ou seja, têm apenas uma consciência situacional, mas também uma compreensão situacional que o leva a modificar conteúdos, estratégias de ensinoaprendizagem e - se necessário - critérios de avaliação, uma vez que a situação extraordinária tem de ser realizada. , além de restrições que não podem ser ignoradas. Neste sentido, em termos de consciência situacional, confirmo que todos os professores poderão estabelecer o sucesso na comunicação com os seus alunos através de diferentes canais e redes, tanto institucionais como pessoais. Da mesma forma, em termos de compreensão situacional, ou ensino de mudanças importantes apontou corpo, dois efeitos da pandemia em uma pessoa, família e vida pessoal para dois alunos, e era sensível à situação antes de confrontar seu papel de professor e fazer ou movimentos necessários para cada criança. adapte-se a uma situação desafiadora em várias neves.

Palavras-chave: compreensão situacional, consciência situacional, ensino superior, emergência sanitária.

Fecha Recepción: Junio 2020

Fecha Aceptación: Diciembre 2020

\section{Introducción}

La pandemia originada por el virus Sars-Cov-2 (covid-19) ha impactado al mundo de diversas formas. Pese a las previsiones que la Organización Mundial de la Salud (OMS) realizó y las recomendaciones que fueron gradualmente endureciéndose para pasar de medidas voluntarias a restrictivas, ningún país del mundo ha podido sopesar por completo el impacto generado en lo económico, social, político, educativo y cultural.

En el caso de México - hasta el momento de escribir el presente documento-, este ha sido uno de los últimos países en vivir las implicaciones de la pandemia, pero ante el escenario mundial fue posible anticiparse con algunas estrategias para aplanar la curva de contagios, aunque hasta ahora solo se tienen posibles previsiones del modelo de vigilancia epidemiológica Centinela, el cual estima contagios lineales, mas no exponenciales. Ante esto, como casi en todo el orbe, la reducción de la movilidad, la 
Revista Iberoamericana

de las Ciencias Sociales y

Humanísticas

ISSN: $2395-7972$

suspensión de actividades no esenciales y el paro de servicios escolares se convirtieron en una realidad. Particularmente en el sureste del país, con una semana de anticipación de las indicaciones nacionales, se decidió suspender toda actividad, primero educativa y social, y posteriormente económica no esencial.

Por tanto, en este trabajo se presenta — a 35 días de la suspensión de labores — la visión de los docentes de una licenciatura en educación en relación con algunos de los retos, facilidades y percepciones de las adaptaciones que han tenido que hacer a su práctica docente, así como las formas en que consideran que los estudiantes han reaccionado a la actual situación de contingencia. La premisa central es que en la medida en que el profesorado logra demostrar altas capacidades para adaptarse, realizar adecuaciones y ser flexibles al cambio se puede influir en la formación de futuros docentes, los cuales necesitan esos referentes para hacer frente a futuros escenarios laborales que posiblemente puedan distar de lo aprendido en las aulas universitarias.

\section{Del docente novel al experto: comprensión y adaptación situacional}

Existen diferentes teorías y propuestas sobre cómo debe formarse a un profesional del área de pedagogía y educación, así como una intensa discusión en cuanto al número de años, prácticas y experiencias que conducen a un profesional de novel o novato a experto. En términos generales, nos apoyaremos en la propuesta teórica de Pérez (1996) y Elliot (1993), quienes sitúan el nivel de pericia del profesorado no necesariamente con los años de experiencia, sino con la posibilidad de transitar hacia una comprensión situacional de su contexto para hacer las adaptaciones que mejor funcionen a las nuevas condiciones.

No obstante, este concepto comprensión situacional generalmente es tomado como sinónimo de conciencia situacional, cuando en el mejor de los casos debería ser el paso siguiente. Casanovas (2016) menciona sobre la comprensión situacional que "en ocasiones se confunde este concepto con el de conciencia situacional (situation awareness), que se limita a barajar elementos físicos del entorno para comprender nuestra situación relativa y no ser sorprendidos por ellos" (párr. 10), es decir, la conciencia situacional hace referencia a nuestras posibilidades de interpretar el contexto físico inmediato y tomar decisiones en función de ello. Al vincularlo con el ámbito de la docencia, podría entenderse este término como la posibilidad del docente de analizar la distribución del salón de clase, el número de estudiantes y su diferenciación por sexo y edad, el horario (matutino, vespertino, nocturno, sabatino) de la asignatura, su tipología (obligatoria, optativa o de libre configuración), el semestre en que está ubicada, su 
Revista Iberoamericana

de las Ciencias Sociales y

Humanísticas

ISSN: $2395-7972$

modalidad (presencial, virtual, mixta), entre otros elementos que configuran el espacio físico (entendido en la más amplia acepción del vocablo) que le permitan tomar decisiones con base en dichas circunstancias.

Valencia (2016) señala que el concepto conciencia situacional fue por primera vez usado en el ámbito militar, particularmente con los pilotos de guerra; sin embargo, la evolución del término a partir de la psicología cognitiva dio lugar a "todo un andamiaje metodológico que permite a los individuos el correcto desempeño de sus actividades en ambientes diversos a fin de evitar cualquier tipo de pérdida" (párr. 5). Por ende, el concepto ha tenido aplicaciones desde la seguridad empresarial, el campo de la criminología y, para propósitos del presente artículo, la educación.

De acuerdo con Cordón, Olivier, García y Walliser (2014), la conciencia situacional (SA, por sus siglas en inglés) se define de la siguiente manera:

Es el proceso de creación y comprensión de un mapa o representación mental del entorno del sujeto, especialmente en sistemas complejos y con sobrecarga estimular, a través de la selección de la información recibida, su posterior elaboración y, finalmente, la toma de decisiones pertinentes (p. 83).

Como ejemplo de lo anterior, haremos referencia al tema en boga: una pandemia. Es posible crear un mapa en relación con los conocimientos al respecto de la contingencia sanitaria y las implicaciones de esta para la familia, la escuela y el sistema de salud; en consecuencia, orientar al respecto de qué lugares evitar, así como qué medidas son pertinentes para no agravar las condiciones, lo que supondría una toma de decisiones razonada al respecto. Sin embargo, la evidencia empírica en países como México, Italia, España, entre otros, demuestra que no fue suficiente para evitar que la población no acatara ciertas restricciones voluntarias (en principio) hasta que fue demasiado tarde, es decir, falló la comprensión situacional de lo que la epidemia podría representar.

De acuerdo con Endsley (1996, citado por Cordón et al., 2014), la SA se desarrolla en tres niveles. El primero es la percepción de los elementos del entorno, vinculado directamente con la posibilidad física de percepción y el almacenamiento "bruto" de cifras sin procesar, aunque algún dato posteriormente pueda ser recuperado para confirmar una variable presente, pero todavía sin integrar a un panorama más amplio de la situación. Es en el segundo nivel donde — si bien todavía no necesariamente hay una comprensión relevante para el desarrollo de una tarea - sí se produce una integración de los datos recopilados, como piezas de información que permitirán determinar su relevancia según los propósitos individuales que cada sujeto tenga. Finalmente, el tercer 
Revista Iberoamericana

de las Ciencias Sociales y

Humanísticas

ISSN: 2395 - 7972

estadio o nivel es la predicción de futuro, estatus en el cual se proyectan escenarios futuros del entorno, por supuesto, con base en la información recopilada e integrada en los niveles 1 y 2 .

Ahora bien, Endsley (2015) también señala que estas etapas se presentan en la SA, no de forma lineal, sino ascendente. Una persona que entiende una situación tiene mejor conciencia situacional que una que puede leer datos en una pantalla, pero no comprende lo que significan. De igual forma, una persona que puede proyectar un futuro plausible y los estados del sistema y del medio ambiente tiene mejor SA que una que no puede.

Por ejemplo, en una situación de aula, durante una explicación del profesor, observar a dos estudiantes levantar la mano implicaría el nivel 1, es decir, la conciencia de que se ha presentado un gesto por dos integrantes del grupo; en el nivel 2, ese gesto y otros elementos como solicitar la palabra por parte de uno de ellos junto con otras señales verbales y no verbales - llevaría a cederle la palabra a uno de ellos (o a ambos, según sea el caso); y en un tercer nivel el predecir que la participación de dichos estudiantes posiblemente sea constante durante el desarrollo de esa y las demás sesiones de clase. Ahora bien, aun con las posibilidades de predicción, en el ámbito de la docencia, ser capaces de predecir si habrá más participaciones no implica juzgar la calidad, intencionalidad, ni la carga emocional, ni lo que posiblemente pueda representar para el participante o para el resto del grupo. Todo ello estaría a nivel de comprensión situacional (CS).

La comprensión situacional "implica discriminar y sintetizar los elementos significativos de la práctica de cualquier situación en un dibujo coherente y unificado de la situación concreta (...), condicionada por los intereses prácticos en la realización de valores profesionales dentro de una situación" (Elliot, 1993, citado por Pérez, 1996, p. 347). Es decir, no solo hay una toma de decisiones, sino juicios de valor desprendidos de la situación y de los significados previos valorados en las decisiones tomadas.

$\mathrm{Al}$ respecto, Pérez (1996) explica que un docente experto es aquel que se apoya en el análisis de la situación concreta, pero más aún en los resultados que se generan de la interacción entre la comprensión y su actuación profesional, elementos que mutuamente se complementan y potencian. En este sentido, y bajo un esquema hermenéutico de la construcción de los significados de la docencia, el profesorado debe ser autónomo en su toma de decisiones porque eso lo conducirá a generar sus propias conclusiones relativas a lo que para él o ella significa ser docente y las mejores prácticas derivadas de esta comprensión. Si nos quedáramos únicamente a nivel de SA, entonces bastaría con prescribir un programa de curso con sus respectivos planes de sesión y 
Revista Iberoamericana

de las Ciencias Sociales y

Humanísticas

ISSN: $2395-7972$

pedirle al profesorado que lo siga al pie de la letra, partiendo de las premisas que tanto el programa de curso como el plan de sesión están elaborados con base en los principios fundamentales de la pedagogía y la didáctica, por lo que el docente será considerado experto en la medida en la que analiza y predice cómo implementar satisfactoriamente esos contenidos en el tiempo destinado para ello.

Entonces, ¿debe o no hacer adecuaciones el profesorado? La respuesta —a nivel de comprensión situacional - es sí, pero bajo el entendimiento de que esas adaptaciones siempre serán relativas y deben atender a otras condiciones sociales, materiales y económicas que trascienden al hecho de no acudir a la escuela por causa de una pandemia; en otras palabras, la emergencia sanitaria y el paro de labores académicas son variables adicionales que se deben considerar para emprender o no ciertos cambios. No es cambiar el curso porque hay una pandemia, sino preguntarse qué implicaciones tiene la pandemia en la sociedad, en la escuela, en la vida de los estudiantes, en la praxis docente y, en última instancia, en los alcances y limitaciones actuales de los cursos que cada docente imparte.

\section{Contexto del estudio}

La facultad en cuestión es la de más reciente creación de la universidad. Esta última tiene 95 años de existencia y se ha posicionado gradualmente como una de las cinco mejores en el sureste del país. Por su parte, la Facultad de Educación fue fundada hace 36 años y tuvo la particularidad de que, a diferencia de otras dependencias de la misma universidad, inició primero con su oferta de posgrado antes que de licenciatura.

Actualmente, oferta tres programas de posgrado (especialización en Docencia y dos maestrías en Investigación Educativa y en Innovación Educativa) y dos programas de licenciatura (en Educación y en Enseñanza del Idioma Inglés); la oferta de la licenciatura en Educación se realiza en dos campus; Mérida (capital del estado de Yucatán) y la Unidad Multidisciplinaria Tizimín, en el oriente de la región (Facultad de Educación de la Universidad Autónoma de Yucatán [FEUADY], 2020).

En sus orígenes, la mencionada facultad reclutó personal de otras escuelas y facultades que dieron un sentido muy particular a sus perfiles de egreso, principalmente porque no pertenecían al área de pedagogía o ciencias de la educación, es decir, prácticamente todo el personal fundador fueron ingenieros, psicólogos, arquitectos, químicos, etc., con especialidad en docencia, pero ninguno pedagogo o egresado de la carrera de magisterio. Es a partir del egreso de la primera generación el momento en el que gradualmente se empiezan a incorporar a la planta docente los primeros licenciados 
Revista Iberoamericana

de las Ciencias Sociales y

Humanísticas

ISSN: $2395-7972$

en Educación. Actualmente, es posible identificar, de manera tácita, tres grandes grupos de profesores: los fundadores (de formaciones diferentes, con más de 25 años de labores y algunos próximos a la jubilación); los profesores consolidados (mayoría de licenciados en Educación, con 15 a 25 años laborando en la DES) y el profesorado de nueva generación (todos egresados de la licenciatura, con experiencia entre 5 y 15 años en sus respectivas áreas).

El perfil de egreso de la licenciatura ha sufrido diferentes modificaciones desde 1984, año en el que el Consejo Universitario de la IES aprobó el primer plan de estudios, aunque básicamente conserva la esencia de sostener la formación en cuatro áreas o campos: administración educativa, currículo, docencia y orientación educativa. Sin embargo, al día de hoy se sigue luchando con la idea de que un egresado o egresada de la carrera únicamente está capacitado(a) para la docencia, cuando el perfil es mucho más amplio. Posiblemente, esto también ha hecho que el profesorado asuma la necesidad de desarrollar en el alumnado competencias diversas que les permitan ampliar sus opciones laborales y académicas, ante una sociedad que — aun con 30 generaciones egresadastodavía no conoce realmente las posibilidades de la licenciatura en Educación.

En ese sentido, hay una conciencia situacional de la que se desprenden también significados a partir de las interpretaciones que se hacen del contexto; de acuerdo con Albercht (2007), "los seres humanos llenan los espacios con significados, y dichos significados influyen en sus interacciones" (párr. 7), es decir, es bidireccional. Ahora bien, precisamente en este artículo el interés recae en conocer qué tanto esas interacciones — que ya tienen una carga simbólica en el espacio presencial — pueden ser adaptadas a las nuevas condiciones que la pandemia trajo consigo.

Estas nuevas condiciones tomaron desprevenido al profesorado debido a decisiones gubernamentales adelantadas, a pesar de que el panorama en otros lugares del orbe ya había evidenciado el tipo de restricciones que los gobiernos respectivos habían implementado como parte del aprendizaje acerca del comportamiento social en torno a la enfermedad.

El gobierno del estado en donde está localizada la universidad decretó con una semana de anticipación al gobierno federal el inicio del paro de labores no esenciales, que además coincidió con un día feriado inhábil oficial, por lo que desde el 17 de marzo se suspendieron las labores académicas (Universidad Autónoma de Yucatán [UADY], 15 de mayo de 2020).

Por tal motivo, el $1 .^{\circ}$ de abril de 2020 la administración escolar solicitó al profesorado avanzar gradualmente en los contenidos de sus asignaturas, previendo — para 
Revista Iberoamericana

de las Ciencias Sociales y

Humanísticas

ISSN: 2395 - 7972

esa fecha- que la situación sanitaria permitiera un regreso en la primera semana de mayo. La indicación fue emplear estrategias que favorezcan el desarrollo exitoso de los contenidos y su aprendizaje efectivo. Para hacer esa solicitud, por su parte, la administración de la dependencia realizó un sondeo de las condiciones tecnológicas y de conexión del alumnado de los dos programas de pregrado que ofrece (Enseñanza del Idioma Inglés y Educación; este último en dos campus: Mérida y Tizimín). La respuesta obtenida indicó que el porcentaje de alumnado que cuenta con las condiciones para cumplir con el avance en las asignaturas era poco más de $75 \%$ en promedio, con particulares diferencias entre las unidades académicas. Por tal motivo, se dejaba a consideración del profesorado los tiempos, formas y estrategias para avanzar con miras al regreso a las aulas hacia finales de abril o principios de mayo. No obstante, el 16 de abril la autoridad nacional y, posteriormente, la estatal anunciaron la ampliación de la jornada denominada Sana Distancia, por lo que se extendió el paro de actividades no esenciales y algunas esenciales. Entonces, el regreso a clases se estimó en ese momento hacia principios de junio, aunque posteriormente se descartó la posibilidad de regresar a la presencialidad educativa.

\section{Objetivo}

Describir las adecuaciones que el profesorado del área de pedagogía y educación ha realizado en sus propuestas e interacciones didácticas a partir de la crisis generada por la covid-19 en una dependencia perteneciente a una universidad del sureste de México.

\section{Metodología y participantes}

Este estudio fue exploratorio, con enfoque mixto, pues se basó en recabar datos de un diseño tipo encuesta y en realizar un análisis de contenido de respuestas abiertas. La importancia de utilizar un enfoque mixto se halla en que permite asegurar la comprensión de un fenómeno determinado desde diferentes ángulos, práctica que se ha vuelto común en campos de diversas disciplinas como las ciencias de la salud (enfermería), así como en las ciencias sociales, humanidades (o ciencias del comportamiento) y económico-administrativas (Tashakkori y Teddlie, 2003). Asimismo, se cataloga como de corte transversal porque, de acuerdo con Hernández, Fernández y Baptista (1991), "los diseños de investigación transeccional o transversal recolectan datos en un solo momento, en un tiempo único. Su propósito es describir variables, y analizar su incidencia e interrelación en un momento dado" (p. 247). 
Revista Iberoamericana

de las Ciencias Sociales y

Humanísticas

ISSN: $2395-7972$

La colecta de datos se realizó justamente en medio de la crisis sanitaria, a partir de un instrumento en línea. La población estuvo constituida por el profesorado de dicha facultad que impartía clases en la licenciatura en Educación (Unidad Mérida) en el semestre de primavera, enero-mayo.

Con esos criterios, el 17 de abril se le envío al profesorado una encuesta constituida por 19 preguntas distribuidas del siguiente modo: datos de identificación (ítems del 1 al 7) con preguntas como el nombre de su curso, el número de estudiantes, la modalidad (regular o acompañamiento), entre otras. De los ítems 8 al 12 se usaron preguntas en escala de Likert para conocer si habían implementado algún tipo de estrategia debido a la situación de emergencia sanitaria; finalmente del ítem 13 (excepto el 15) al 19 se emplearon preguntas abiertas relacionadas con detalles más específicos sobre las adecuaciones, retos y sugerencias que el profesorado pudiera hacer en torno a la eventualidad. A continuación, se presentan los principales resultados.

\section{Resultados}

Actualmente, se imparten 61 cursos en el programa educativo (Unidad Mérida), entre asignaturas obligatorias, electivas o de libre configuración. Cabe señalar que dadas las características de flexibilidad del plan de estudios, para el caso de las asignaturas obligatorias, estas son impartidas por dos profesores, cada uno en distintos horarios, de tal forma que el estudiantado pueda seleccionar al profesor-horario que mejor se adapte a las necesidades de su trayecto formativo.

En ese sentido, de los 61 cursos en total, se tuvo una participación de $93.84 \%$ del profesorado, es decir, hubo una excelente respuesta de los docentes. Ahora bien, los cursos pueden ser de forma regular (primera vez que el estudiante se matricula en la asignatura) o por acompañamiento ${ }^{1}$ (UADY, 2012). Ambas modalidades vienen a colación debido a que del porcentaje de respuestas, $9.8 \%$ fueron cursos por acompañamiento y $90.2 \%$ fueron de forma regular.

Después de los ítems relacionados con los datos demográficos, en el ítem 8 se le preguntó al profesorado sobre el avance en sus cursos; los resultados se muestran en la figura 1.

\footnotetext{
${ }^{1}$ Modalidad que sustituye a los exámenes extraordinarios y que en el modelo educativo actual de la universidad parte de la premisa de un diagnóstico de las áreas de oportunidad que no le permitieron al estudiante acreditar satisfactoriamente la asignatura; en consecuencia, se diseña un plan específico por estudiante, con las ventajas y retos que esto implica y que escapan del propósito de este texto.
} 
Revista Iberoamericana de las Ciencias Sociales y Humanísticas

ISSN: $2395-7972$

Figura 1. Porcentaje de cursos con actividad virtual (LE Mérida)

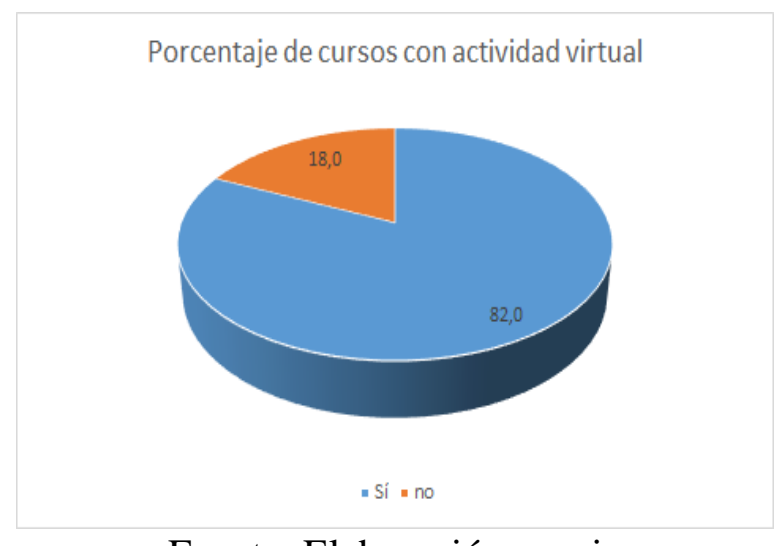

Fuente: Elaboración propia

Como puede observarse en la figura anterior, es mínima la proporción de profesores que, al momento de la encuesta, no habían tenido algún tipo de contacto o acercamiento a través de los medios tecnológicos a su alcance. Posteriormente, en otro de los cuestionamientos se indagó acerca de qué tanto consideraban que se había avanzado durante este período para cubrir los contenidos y desarrollar las competencias esperadas del curso.

Figura 2. Porcentaje de avance de cursos (LE Mérida)

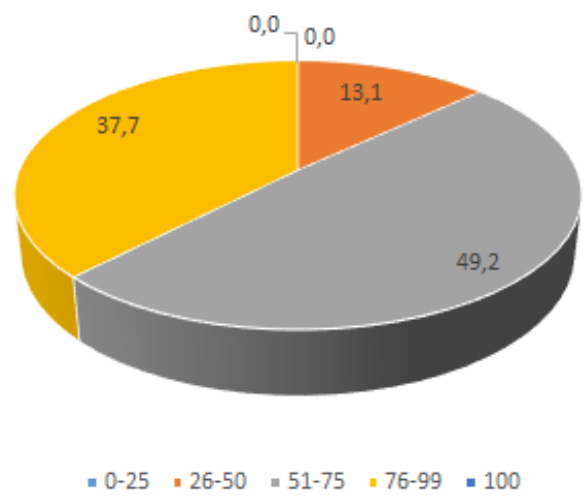

Fuente: Elaboración propia

Básicamente, la mitad de los cursos presentó un avance entre $51 \%$ y $75 \%$, seguido por un avance sustancial al menos de $76 \%$ en adelante, y aunque ninguno manifestó haber concluido su curso, tampoco la evidencia recolectada indica grados mínimos de avance (entre $0 \%$ y $25 \%$ ). Esto hace eco con la figura anterior, en términos de que el profesorado había continuado —en sus posibilidades - avanzando con sus cursos, tratando de respetar la variedad y demandas propias de cada asignatura.

Posteriormente, se les cuestionó sobre la herramienta tecnológica más empleada para mantener el vínculo con los estudiantes; los resultados se presentan a continuación: 
Revista Iberoamericana

de las Ciencias Sociales y

Humanísticas

ISSN: $2395-7972$

Figura 3. Plataformas o medios virtuales empleados

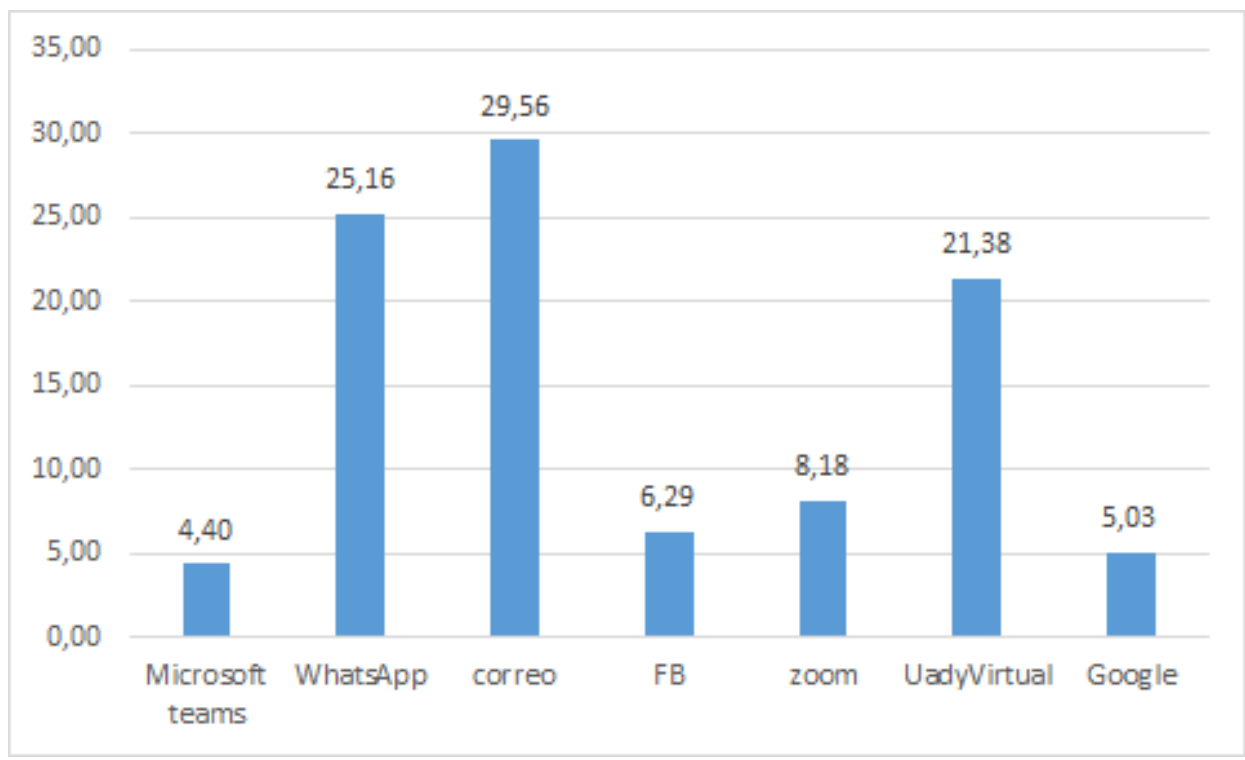

Fuente: elaboración propia

Como puede apreciarse, se les dio la oportunidad de seleccionar más de un medio o plataforma y se les permitió sugerir alguna que no estuviera en el listado de opciones. De esta figura destaca que el principal medio usado es el correo electrónico, que podría considerarse la opción más tradicional en el abanico de opciones tecnológicas actuales. El segundo lugar lo ocupó la mensajería instantánea (WhatsApp) y en un tercer lugar el sistema de gestión del aprendizaje institucional, denominado UADY Virtual, el cual debería encabezar la lista dado que desde la implementación del Modelo Educativo para la Formación Integral (MEFI) en 2012 en cada dependencia se han ofrecido una serie de capacitaciones para el profesorado, que van desde cursos de planeación en ambientes no convencionales hasta el uso de herramientas específicas para configurar la plataforma.

Otra pregunta planteada tuvo que ver con qué tan favorable consideraba la respuesta del estudiantado a trabajar en ambientes no convencionales.

Figura 4. Respuesta del estudiantado

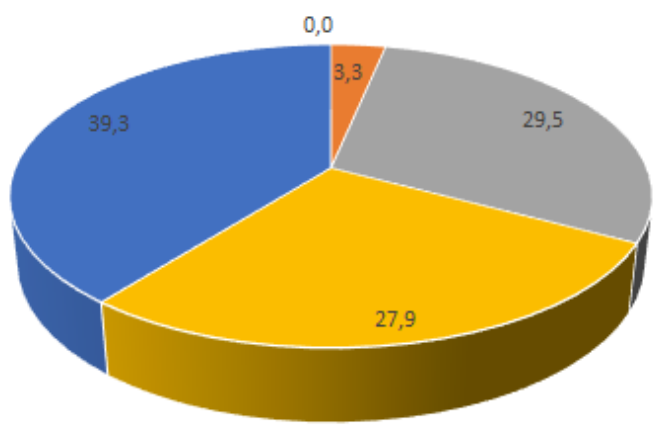

- $1.2 \cdot 3 \cdot 4 \cdot 5$

Fuente: Elaboración propia 
Revista Iberoamericana

de las Ciencias Sociales y

Humanísticas

ISSN: $2395-7972$

En una escala de Likert (siendo 1 la respuesta nula y 5 excelente), el promedio considerado por el profesorado fue de $\mathrm{x}=4.01 \mathrm{y}$ la mediana fue de 4 , por lo que hay consenso en los datos, es decir, se percibe una respuesta muy buena por parte del alumnado. Hasta esta sección descriptiva únicamente podríamos inferir que el profesorado tiene una conciencia situacional de lo que está ocurriendo, lo que hace posible mapear el fenómeno que se está viviendo y tomar decisiones con base en ello, como continuar la implementación de sus cursos a través de medios no convencionales y personales (como el número de móvil y el uso de WhatsApp). En este punto, no se encontraron diferencias importantes en estas estrategias en relación con variables como sexo, antigüedad o tipo de curso.

Ahora bien, ya que se percibe una conciencia situacional, surge la siguiente interrogante: ¿es posible que el profesorado haga cambios de fondo para el cierre de sus cursos? Para ello, se procedió al análisis cualitativo de las respuestas abiertas a través de la técnica de lápiz y papel. De este modo se categorizaron las respuestas del profesorado en las preguntas $13,14,16,17,18$ y 19. En el caso de la pregunta 15 no fue tomada en cuenta para el análisis cualitativo debido a que se refería a la percepción de los docentes acerca de la respuesta del alumnado. Para efectos de lo anterior, se preguntó al profesorado cuáles eran las dificultades a las que se había enfrentado para la implementación de clases a distancia. Anticipadamente, estas se habían dividido en dos categorías: 1) aquellas de tipo técnico, tales como el equipamiento, la conectividad y el tipo o naturaleza del curso y 2) aquellas relativas a lo humano, como las características personales, los valores, etc.

De acuerdo con la información obtenida, las dificultades de tipo técnico fueron las siguientes:

- Baja calidad del servicio de internet contratado tanto por docentes como por estudiantes.

- La eficiencia del servicio de energía.

- No contar con acceso a los servicios de internet (alumnado y profesorado).

- Falta de equipamiento (alumnado y profesorado).

- Falta de capacitación docente para impartir clases en modalidad virtual.

- Desconocimiento del uso de aplicaciones para tomar las clases a distancia o no contar con los programas necesarios para las clases.

- Falta de apoyo del personal experto en tecnologías.

- El tiempo que se invierte en la planeación del trabajo a distancia.

- Naturaleza del curso (predominantemente teórico o predominantemente práctico). 
Revista Iberoamericana de las Ciencias Sociales y Humanísticas

ISSN: $2395-7972$

A continuación, se citan algunos de los comentarios que ilustran las razones anteriores:

- Velocidad de Internet, falta de equipo para grabar videos.

- He notado que hay estudiantes que no cuentan con las herramientas tecnológicas necesarias para continuar con todas las actividades desde su casa, es una preocupación que pueda verse afectado su avance y posibilidades de éxito.

- Mis mayores dificultades son técnicas que supero a partir de lectura y revisión de videotutoriales para programar actividades diferentes, como son programación de videoconferencias, clases pregrabadas o elaboración de videos, al igual que cuestiones de Moodle, que [sic] aunque tomé el curso que nos dio la... he tenido que repasar con tutoriales. Me preocupa que todos estén y tengan las mismas condiciones para finalizar el curso, me refiero al internet, al uso de herramientas tecnológicas y al uso de la plataforma.

En el mismo sentido, las dificultades de tipo humano encontradas se citan a continuación:

- La responsabilidad del estudiantado al no buscar la forma de ponerse en contacto con los docentes o con los miembros del equipo.

- La responsabilidad del profesorado para dar seguimiento a los estudiantes que se encontraban desarrollando tesis.

- La resiliencia o su falta para que el estudiantado enfrente la pandemia.

- Falta de motivación del estudiante para el trabajo a distancia.

Los siguientes comentarios son un ejemplo de lo anterior:

- Dificultad para contactar a todos los alumnos y su participación en las actividades grupales e individuales.

- Ninguna, solo un poco la lentitud de respuestas de los alumnos, pero es obvio, están ocupados en asuntos de relevancia familiar, como me han mencionado cuando logran comunicarse, pero van bien.

- También los asesores de los trabajos de tesis no han atendido a los estudiantes en la revisión de sus avances.

Por otro lado, sobresalen las condiciones socioeconómicas familiares del alumnado que dificultaban el pago del internet; de hecho, en algunos casos no se contaba con dicho servicio porque en el lugar de residencia no existía infraestructura tecnológica. Esta fue una categoría emergente que no se incluyó en las previas. 
Revista Iberoamericana

de las Ciencias Sociales y

Humanísticas

ISSN: $2395-7972$

Finalmente, varios docentes comentaron que no se habían encontrado con dificultades de ningún tipo al momento de la encuesta.

En cuanto a la forma en que los docentes habían superado las dificultades anteriores, las respuestas fueron categorizadas en 1) adaptaciones curriculares, 2) aprendizaje, 3) inversión de recursos personales, de tiempo, materiales y financieros, y 4) canales de comunicación.

Sobre las adaptaciones curriculares de los docentes para superar las dificultades, se encontró lo siguiente:

- Ampliar los plazos de entrega.

- Modificar las actividades de aprendizaje.

- Reestructurar la evaluación del proceso y del producto.

- Ofrecer alternativas para suplir la falta de práctica en escenarios reales.

- Elaborar materiales para descargar de manera offline.

El siguiente comentario evidencia el tipo de adaptación curricular llevada a cabo:

- Las dificultades que se han presentado se han abordado tratando de ser más flexible en cuanto al tiempo de entrega de algunas actividades de aprendizaje. Se ha realizado un seguimiento de las sesiones en línea por medio del Facebook, con el fin de mantener informados a los estudiantes que hayan enfrentado alguna dificultad durante las sesiones en línea o para conectarse a las mismas.

En cuanto al aprendizaje, las respuestas demuestran que pudo ser de dos tipos: autónomo y a través de terceros:

\section{Autónomo}

- Buscar tutoriales en YouTube para aprender a usar las herramientas tecnológicas.

- Examinar información para conocer nuevas herramientas para utilizar en el curso.

A través de terceros

- Llevar un curso ofrecido por la universidad y por la empresa de tecnología con la que se tenía convenio.

- Del personal de tecnologías de la dependencia.

- De familiares que dominaban los programas y aplicaciones para la educación virtual.

A continuación, se cita uno de los comentarios referente al aprendizaje que tiene lugar para atenuar los efectos de la pandemia en la docencia: 
Revista Iberoamericana de las Ciencias Sociales y Humanísticas

ISSN: $2395-7972$

- A través de tutoriales y llamado de teléfono a algunos colegas para que me asesoren.

La inversión de recursos personales, financieros, tiempo y materiales se manifestó del siguiente modo:

- Paciencia, mucha paciencia.

- Dedicar más tiempo al rediseño de tareas.

- Comprar artículos tecnológicos para apoyar el trabajo docente.

- Contratar a otra compañía para tener acceso a internet.

De igual forma, se cita uno de los comentarios que hace alusión a alguna de las razones:

- Estoy modificando las actividades de aprendizaje con la finalidad de obtener otro tipo de ejercicio donde se puedan aplicar los contenidos de la asignatura.

Por otra parte, se destaca la preocupación por utilizar diversos medios para comunicarse con el alumnado. Para hacer frente a ello el profesorado recurrió a lo siguiente:

- Conformó grupos de WhatsApp.

- Utilizó sus perfiles personales de Facebook para la transmisión en vivo de las clases.

- Solicitó al área de tecnologías que se activaran las cuentas institucionales de estudiantes de sus cursos.

- Utilizó llamadas telefónicas desde sus números celulares personales.

- Se apoyó en los líderes de grupo para compartir información vinculada con sus cursos.

Uno de los docentes hizo referencia a lo anterior a través del siguiente comentario:

- Se ha realizado un seguimiento de las sesiones en línea por medio del Facebook con el fin de mantener informados a los estudiantes que hayan enfrentado alguna dificultad durante las sesiones en línea o para conectarse a las mismas. Otra vía de información ha sido el Whatsapp para consultar cualquier duda que se pudiera presentar por medio de la vocal del grupo.

En congruencia con la pregunta anterior, aquellos(as) docentes que no reportaron dificultades ante la impartición de clases en el contexto pandémico también respondieron que no habían tenido necesidad de hacer cambios de ningún tipo. 
Revista Iberoamericana

de las Ciencias Sociales y

Humanísticas

ISSN: $2395-7972$

Asimismo, se indagó en el profesorado acerca de las adaptaciones llevadas a cabo para la docencia a distancia, las cuales tienen que ver con 1) contenidos y sus desagregados, 2) estrategias de enseñanza-aprendizaje, 3) estrategias de evaluación y 4) medios y recursos didácticos.

Ahora bien, en cuanto a las adaptaciones de contenido y sus desagregados, se encontró que el profesorado efectuó lo siguiente:

- Revisó contenidos más enfocados en lo teórico.

- Redujo el contenido revisado en algunas unidades y lo enfocó en lo más relevante.

- Reorganizó contenidos.

El siguiente comentario refleja lo anterior:

- Los contenidos se [sic] faltan se revisarán de modo conceptual y a través de ADAS. Aun cuando quisiera y pudiera plantear un seminario más, mi preocupación es en cuanto a las estudiantes que no tengan disponibilidad de acceso al internet. Estoy en la adaptación de las ADAS a fin de que no dependan al $100 \%$ de los casos reales para resolverlas.

Como era esperado, entre las adaptaciones con mayor mención estuvieron aquellas relacionadas con las estrategias de enseñanza-aprendizaje, tales como:

- Utilizar REAS.

- Buscar alternativas para no depender de los escenarios reales, tales como videos, entrevistas y documentales.

- Utilizar las asesorías como apoyo para el trabajo en grupos colaborativos.

- Apoyarse en cursos online gratuitos para que los estudiantes tomaran como alternativa a la práctica o para complementar sus conocimientos.

- Escanear los materiales de lectura.

- Grabar videos explicando el tema y publicarlos en YouTube.

- Aumentar la cantidad de lecturas (estudio independiente).

- Disminuir el porcentaje de actividades en grupo para dar lugar al aprendizaje individual.

- Desglosar las actividades de aprendizaje lo mejor posible para que fueran entendibles sin la presencia inmediata del profesor.

Una de las profesoras refirió lo siguiente:

- Se hará un cambio en la unidad de información financiera, cambiando los ejercicios por un curso en línea de Coursera: Contabilidad para no contadores, gratuito. Con base en este se desarrollarán foros de discusión 
Revista Iberoamericana de las Ciencias Sociales y Humanísticas

ISSN: $2395-7972$ enfocados a determinar cómo la información financiera apoya a la organización en la gestión y toma de decisiones.

En cuanto a las adaptaciones de las estrategias de evaluación, se encontraron las siguientes:

- Ajustes en la rúbrica de calificación y rediseño de instrumentos.

- Eliminar productos tangibles.

- Utilizar aplicaciones para evaluar, como Active Presenter, Google Hangouts, WhatsApp o Forms.

- Utilizar Google Drive para evaluar los avances, aunque la versión final se debía subir a la plataforma institucional.

- Disminuir el número de prácticas evaluables.

- Cambiar las características del trabajo final.

El siguiente comentario es una muestra de las razones anteriores:

- En la evaluación final, en donde la competencia a desarrollar se hace evidente mediante la visita de campo a su unidad receptora, las visitas de campo estaban programadas para esta semana, del 20 al 26 de mayo; por eso, se van a adecuar los contenidos y criterios con que se evaluará la competencia de la asignatura, lo más probable es que se quede limitado a un aspecto conceptual o declarativo.

Asimismo, en este apartado se destacó con varias menciones la adaptación de los medios y recursos empleados para la enseñanza:

- Utilizar más el correo electrónico.

- Utilizar aplicaciones como Zoom, WhatsApp, Zoom, Google Classroom.

- Permitir la entrega de tareas por diversas plataformas.

- Utilizar videos en streaming.

El siguiente comentario hace referencia a la diversificación de los medios utilizados para continuar con su docencia:

- Hasta al momento, sólo se ha cambiado las fechas y la forma de revisar avances, ya sea por WhatsApp o correo electrónico, y se agregaron foros para compartir los temas y presentar las conclusiones de sus aprendizajes.

Por otro lado, y ante la preocupación por parte de las autoridades universitarias de enfrentar un notable índice de reprobación ante escenarios desiguales que no permitieran a un porcentaje de estudiantes responder exitosamente a los requerimientos académicos derivados de sus asignaturas, se buscó conocer con mayor detalle aquellas adaptaciones referidas a la evaluación. En tal sentido, la información obtenida se categorizó en 
Revista Iberoamericana

de las Ciencias Sociales y

Humanísticas

ISSN: $2395-7972$

adaptaciones relacionadas con 1) criterios evaluables, 2) valor o puntaje asignado, 3) tiempo y 4) medios empleados.

En cuanto a las adaptaciones de criterios evaluables, los docentes mencionaron que realizaron lo siguiente:

- Ajustar las rúbricas o los instrumentos evaluables.

- Integrar actividades de aprendizaje para disminuir su número.

- Adecuar pruebas de desempeño.

- Implementar alternativas evaluables para sustituir aquellas relacionadas con escenarios reales o prácticas de campo.

- Considerar las vivencias durante la contingencia como criterio a evaluar.

- Trabajar con los datos que se cuentan al momento de la fecha de corte para llevar a cabo el análisis de la información (en el caso de las tesis en fase de recolección de datos).

El siguiente comentario se refiere a incluir las vivencias respecto de la contingencia en la evaluación final:

- Hice un corte de lo avanzado y rediseñé tareas más factibles de realizar con base en su [sic] posibilidades reales. La nueva tarea se orienta al rescate y puntualización de sus experiencias y vivencias ante la contingencia.

En cuanto al valor asignado de manera inicial en las planeaciones didácticas a los diferentes criterios evaluables, el profesorado mencionó lo siguiente:

- Ajustar el valor en los productos a entregar.

- Dar más valor a lo teórico.

- Ajustar el valor de acuerdo con las estrategias empleadas por la cuarentena.

- Evitar penalizar la falta de práctica.

Uno de los comentarios que hacen evidente lo anterior es el siguiente:

- Se han ajustado los puntajes con el fin de promediar las actividades que hasta el momento se han podido realizar con las sesiones en línea y los avances en la preparación de materiales y las planeaciones de clase que los practicantes ya han elaborado. Esto se ha decidido en consenso con el grupo en donde todos estuvieron de acuerdo. Esta decisión no tiene una repercusión directa en los promedios de los estudiantes, ya que no se les va a penalizar la falta de práctica docente y, en su lugar, van a entregar todos los materiales que han elaborado. 
Revista Iberoamericana

de las Ciencias Sociales y

Humanísticas

ISSN: $2395-7972$

Por supuesto, también fueron constantes las respuestas relativas a la ampliación de los plazos de entrega, tomando en cuenta las condiciones particulares de cada uno de los estudiantes.

En cuanto a los medios empleados para llevar a cabo la evaluación, al igual que los considerados para la docencia, el profesorado mencionó que estaba dispuesto a utilizar los oficiales (aula virtual, correo electrónico, Zoom, Teams, Google Classroom, WhatsApp, etc.) para llevar a cabo esta actividad.

Cabe resaltar que algunas respuestas evidenciaron la espera de las disposiciones oficiales para determinar cuáles serían los cambios respecto a la evaluación de sus cursos.

Finalmente, fue importante conocer qué proponía el profesorado — dada su experiencia, conocimiento disciplinar y relación con el alumnado- para concluir el periodo escolar. Las respuestas obtenidas se categorizaron en 1) educación a distancia, 2) conciencia social y 3 ) adaptaciones.

En cuanto a la educación a distancia, el profesorado comentó que era preferible finalizar de ese modo, es decir, utilizando los medios y recursos disponibles para ello. Mención especial merecen las respuestas obtenidas que permitieron dar cuenta del alto nivel de comprensión por parte del profesorado de las diferentes condiciones vividas por cada estudiante, tal como reflejan las siguientes propuestas:

- Ser consciente de las condiciones desiguales del alumnado en relación con la tecnología (conectividad y equipamiento).

- Concluir con lo que se haya visto hasta el momento de cierre de periodo.

- Mantener la cercanía con los estudiantes.

- Permitir la presencialidad a quienes no tuvieran las mismas condiciones de conectividad y equipamiento.

- Contar con mayor apoyo por parte de los asesores para que los estudiantes concluyan las tesis.

- Flexibilizar lo que se espera en cada caso (cada estudiante).

- Permitir al estudiante concentrarse en las experiencias familiares que le acontecen durante la pandemia y considerar que los contenidos pueden verse en el otro semestre.

En concordancia con las propuestas anteriores, se cita la siguiente respuesta:

- Pues no estoy negada al empleo de medios virtuales para finalizar, pero no sé qué tan equitativo sería considerando el caso de los alumnos que no tienen acceso a internet. Me parece que sería conveniente avanzar con los 
Revista Iberoamericana

de las Ciencias Sociales y

Humanísticas

ISSN: 2395 - 7972

que tienen acceso a internet y cuando se dé la reincorporación permitir un periodo de regularización para los demás.

Por último, acerca de las adecuaciones propuestas, se categorizaron del siguiente modo: a) de tiempo, b) de producto final, c) de "enganche", d) de contenidos y e) administrativas. Las respuestas se agruparon como siguen:

- De tiempo: Referidas a ampliar el semestre, eliminando el periodo de verano o intersemestral y abarcando un poco del siguiente.

- De producto final: Relativas a ajustar las características del producto final a partir de los contenidos revisados, la ausencia de la parte práctica y los medios disponibles para llevarlo a cabo.

- De "enganche": Permitir a los estudiantes reconectarse con el curso a través del desarrollo de estrategias innovadoras o de interés para ellos.

- De contenidos: Considerar que si no es importante, no se vea, y si es importante y no puede revisarse, incluirlo en el semestre que sigue (caso de las asignaturas seriadas).

- Administrativas: Aquellas relativas a la flexibilidad del sistema institucional de calificaciones y de las personas a cargo.

$\mathrm{Al}$ igual que en los casos anteriores, se muestran los siguientes comentarios como ejemplo de las propuestas para finalizar los cursos:

- Continuar trabajando como lo veníamos haciendo en línea, o bien continuar con los cursos en el mes de junio para concluir en el mes de julio antes de las vacaciones y eliminar los cursos de verano.

- Se debe adaptar lo que resta del programa a las condiciones de los estudiantes, dándoles oportunidad de seguir, pero a su ritmo y condiciones. Habrá estudiantes que no tengan alguna forma de seguir por medios virtuales, porque carecen de internet y/o computadora en sus domicilios. En estos casos tendría que permitírseles regularizarse al final de la pandemia, dándoles un tiempo proporcional para ello y mediante actividades equivalentes. 
Revista Iberoamericana

de las Ciencias Sociales y

Humanísticas

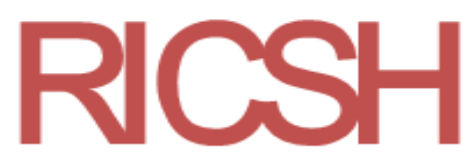

ISSN: $2395-7972$

\section{Discusión}

\section{Adaptaciones y comprensión situacional}

De acuerdo con lo presentado en la sección teórica de este artículo, la diferencia entre conciencia y comprensión situacional radica, entre otros elementos, en el hecho de entender a profundidad el fenómeno y tomar decisiones con base en las consecuencias positivas y negativas. Es decir, la comprensión implica la conciencia, aunque va más allá de ella. La premisa que se discute en el presente texto es si ante la emergencia sanitaria el profesorado debe realizar cambios en su práctica docente.

Ahora bien, aunque esto es evidente, cabe preguntar no solo si dichos cambios obedecen a una conciencia situacional o alcanzan niveles de comprensión situacional, sino también si las diferencias entre docentes con menos años de experiencia y otros más consolidados representan adaptaciones más profundas en la implementación de sus cursos. Esto en concordancia con lo que afirman Metzdorff y Safranchik (2017), quienes apuntan lo siguiente:

El saber práctico de los docentes, como construcción personal y situada en un contexto específico, pone de manifiesto los límites de las teorías didácticas y la especificidad de la práctica docente como ámbito de construcción de la identidad profesional y del saber pedagógico (p. 205).

Es decir, la comprensión situacional pone a prueba, en el docente experto, más allá de lo que usualmente domina de su asignatura en términos de contenidos, estrategias, formas de evaluar, etc., parte de su propia identidad, la refleja, la confronta, la transforma. De alguna manera, implica situarse en el rol de un estudiantado cuyas características generacionales muy posiblemente presenten brechas importantes en comparación con lo que él o ella como docente vivió en su formación universitaria no solo en términos de maneras de interacción con sus pares y docentes, sino también en cuanto al nivel socioeconómico, lugar de origen y residencia, entre otros.

En ese sentido, como pudo apreciarse en las respuestas, el profesorado no solo es capaz de evaluar la situación y proponer adaptaciones de forma. En otras palabras, no se conformaron con trasladar la sincronicidad a otras plataformas como Classroom, Zoom, Microsoft Teams, Google Hangouts y Meet, sino que también adaptaron las estrategias para elaborar nuevos materiales de enseñanza.

Ciertamente no fue así para todos, pues algunos profesores se encontraron con dificultades técnicas que, de alguna manera, los confrontaron con las formas en las que acostumbraban a impartir sus clases; algunos señalaron que definitivamente era la primera vez que vivían un proceso similar; no obstante, lo que hay que destacar es la 
Revista Iberoamericana

de las Ciencias Sociales y

Humanísticas

ISSN: $2395-7972$

flexibilidad de entender que no se trataba de tener sesiones de videoconferencia, sino de ampliar el abanico de posibilidades tanto para ellos como para sus estudiantes. Curiosamente, en el momento de la administración de la encuesta todavía se conservaba la idea de que la duración de la pandemia sería por un par de semanas y que, en definitiva, en el ciclo escolar siguiente, todo regresaría a la presencialidad acostumbrada.

Por lo anterior, es importante destacar que algunos profesores veían en esta consciencia situacional que esto no sería así — ni para ellos ni para sus estudiantes — por lo que optaron por incorporarse a programas de capacitación, ya fueran ofrecidos por la propia universidad o tomados por cuenta propia para enfrentar satisfactoriamente el reto venidero.

Ahora bien, esto no solo impacta en las decisiones mediatas, sino en la formación misma de los estudiantes para profesor, pues perciben la conciencia situacional de su profesorado en acciones directas para la adecuación de la práctica docente. En ese sentido, Sánchez y Jara (2018a) indican que los estudiantes que se forman para profesores viven la incorporación al aula desde una percepción situacional reducida y con esquemas de actuación aún incompletos, los cuales "no aparecen de la nada con el inicio de la enseñanza, sino que se van conformando a través de las experiencias prácticas, preformativas y de formación inicial” (Correa, 2008, citado por Sánchez y Jara, 2018a, p. 251); de hecho, se configuran, preferentemente, durante la etapa inicial de inserción a la tarea docente.

Así, el estudiantado concibe cómo sus profesores lograron superar una situación que, eventualmente, podrá ser insumo de su propia comprensión situacional. Ahora bien, cabe preguntarse si estas adecuaciones realizadas por el profesorado de forma directa o indirecta enseñan comprensión situacional a los estudiantes; es decir, ¿es posible enseñar la comprensión situacional? Si tomamos como paralelo el desarrollo de otras destrezas como la autorregulación y particularmente el pensamiento crítico, esto sí es posible. De acuerdo con Tamayo, Zona y Loaiza (2015):

Lograr avanzar en la formación de pensamiento crítico en los estudiantes necesariamente se articula con las prácticas pedagógicas y la perspectiva didáctica desde las cuales pensamos y hacemos la enseñanza. En otras palabras, es a partir del actuar del maestro en su contexto de aula como se puede incidir en el desarrollo del pensamiento crítico en los estudiantes (p. 114). 
Revista Iberoamericana

de las Ciencias Sociales y

Humanísticas

ISSN: 2395 - 7972

Por ello, es doblemente importante lo que el profesorado está enseñando hoy, pues tendrá repercusiones positivas en la formación personal y profesional del estudiantado, ya que está modelando con sus conductas una habilidad que, de otra forma, quizás hubiera llevado años de práctica para el alumno en formación. Sin embargo, vale acotar que también sería interesante indagar si el profesor hace explícito cómo estos cambios impactan más allá del contenido de la asignatura en sus alumnos, pues la intención es trascender de una formación de profesores bajo un paradigma más tecnocrático hacia uno más de corte reflexivo. En palabras de Fullan y Hargreaves (1996, citados por Sánchez y Jara, 2018b):

El profesorado en formación experimenta la inserción a la práctica y al trabajo docente partiendo de la base de que "el rol de las teorías, las prescripciones externas y las experiencias de otros docentes adquiere relevancia cuando ellas pueden articularse con la experiencia del docente y, a la vez, tenga sentido en su trabajo" (p. 2218).

Es decir, todo lo que los profesores dicen y enseñan al estudiante en formación cobra sentido cuando este ve cómo ellos mismos resuelven problemas, muestran empatía, adaptan su práctica, se actualizan o hacen todos los intentos posibles por responder satisfactoriamente a la situación.

En definitiva, de toda esta experiencia tan retadora y compleja como la generada por la pandemia, y más allá de las implicaciones tecnológicas, es preciso reconocer que ha surgido una alternancia entre el análisis en aula y la formación real directa, de tal manera que se ha permitido valorar (y revalorar) la toma de conciencia del profesor en formación (Carbonneau y Hetú, 2010).

\section{Conclusión}

Los retos que enfrenta el profesorado encuestado se asocian con ceder gradualmente el control de algunos elementos de la clase, como la secuenciación de contenidos, los criterios de evaluación establecidos en el programa y la participación activa y constante del alumnado, puesto que la formación de muchos de ellos se basa justamente en estructurar cada momento del proceso de enseñanza-aprendizaje. Esto, sin embargo, exige repensar la idea de la sincronicidad como un simple sustituto de la sesión presencial, por lo que se deben promover esquemas auténticamente de educación virtual.

En cuanto a las fortalezas, se destaca un entendimiento de la pandemia como una situación atípica que no solo enfrentan los estudiantes, sino también sus familias, lo cual afecta en lo académico, lo personal, lo económico y lo social. Por ende, puede concluirse 
Revista Iberoamericana

de las Ciencias Sociales y

Humanísticas

ISSN: $2395-7972$

que sí hay tanto consciencia como comprensión situacional sobre lo que actualmente ha implicado para todos la contingencia sanitaria y sobre el nuevo significado que adoptarán vocablos como enseñar, aprender y, en general, educar.

\section{Futuras líneas de investigación}

Algunas de las líneas de investigación futuras que de la presente propuesta se derivan tienen que ver con qué tan explícita ha sido esta comprensión situacional del profesorado y qué adaptaciones se realizarán a las adecuaciones de emergencia suscitadas.

Asimismo, sería preciso conocer la opinión del estudiante sobre cómo ha percibido las adecuaciones realizadas por el profesorado, qué nuevos retos $\mathrm{y}$ oportunidades han traído consigo y cómo el estudiante para profesor ha cobrado tanto conciencia como comprensión situacional de la docencia en tiempos de emergencia sanitaria.

De igual importancia resulta analizar tanto la conciencia como la comprensión situacional de los equipos directivos que han afrontado la pandemia, pues posiblemente también han tenido que repensar sus esquemas de cómo funciona la administración educativa en términos de la atención a estudiantes, profesores y padres de familia, así como la supervisión, seguimiento y cumplimiento de la normativa institucional.

Finalmente, en la medida en la que se consolide la formación del profesorado, esto impactará en una mejor educación para las generaciones futuras. 
Revista Iberoamericana

de las Ciencias Sociales y

Humanísticas

ISSN: $2395-7972$

\section{Referencias}

Albretch, K. (2007). Social Intelligence. The new science of success. México: Ediciones B.

Carbonneau, M. y Hétu, J. (2010). La formación práctica de los maestros y el nacimiento de una inteligencia profesional. En Paquay, L. Altet, M. Charlier, E. y Perrenoud, P. (coords.), La formación profesional del maestro. Estrategias y competencias (pp. 107-138). México: FCE.

Casanovas, A. (2016) La comprensión situacional en Compliance. Recuperado de https://www.tendencias.kpmg.es/2016/11/la-comprension-situacional-encompliance/

Cordón, J, Olivier, P., García, M. y Walliser, J. (2014). Diseño y validación de una prueba de selección para controladores de tráfico marítimo basada en la medida de la conciencia situacional. Journal of Work and Organizational Psychology, 30(2), 83-93, Doi: http://dx.doi.org/10.1016/j.rpto.2014.06.002

Elliot, J. (1993). El cambio educativo desde la investigación-acción. España: Morata.

Endsley, M. (2015). Situation Awareness Misconceptions and Misunderstandings. Journal of Cognitive Engineering and Decision Making, 9(1), 4-32. Doi: $10.1177 / 1555343415572631$

Facultad de Educación de la Universidad Autónoma de Yucatán [FEUADY] (2020). Historia de la Facultad de Educación. México. Recuperado de https://www.educacion.uady.mx/index.php?seccion=historia

Hernández, R., Fernández, C. y Baptista, P. (1991). Metodología de la investigación. México: McGraw-Hill.

Pérez, A. (1996). Autonomía profesional del docente y control democrático de la práctica educativa. En Manzano, P. (coord.) (1996), Volver a pensar la educación (vol. II) (pp. 339-353). España: Morata.

Metzdorff, V. y Safranchik, G. (2017). El vínculo entre principiantes y expertos en el proceso de inmersión en la práctica. Experiencia en un Instituto de Formación Docente en Argentina. Revista Entramados, Educación y Sociedad, (4). 201-211.

Sánchez, G. y Jara, X. (2018a). Habilidades profesionales asociadas a la docencia. Fijando posiciones de profesores en formación. Sophia Austral, (22), 247-269.

Sánchez, G. y Jara, X. (2018b). De la formación inicial al trabajo docente: comprensión de la trayectoria. Revista Educación. 42(2). Doi: https://doi.org/10.15517/revedu.v42i2.23996 
Revista Iberoamericana

de las Ciencias Sociales y

Humanísticas

ISSN: $2395-7972$

Tamayo, O., Zona, R. y Loaiza, Y. (2015). El pensamiento crítico en la educación. Algunas categorías centrales en su estudio. Revista Latinoamericana de Estudios Educativos, 11(2), 111-133. Recuperado de https://www.redalyc.org/articulo.oa?id=1341/134146842006

Universidad Autónoma de Yucatán [UADY] (2012). Modelo educativo para la formación integral. México: UADY.

Universidad Autónoma de Yucatán [UADY] (15 de mayo de 2020). Comunicado: suspensión de actividades presenciales. México: UADY. Recuperado de https://www.uady.mx/noticia/comunicado-suspension-de-actividadespresenciales-uady

Tashakkori, A. y Teddlie, C. (eds.) (2003). Handbook of Mixed Methods in Social \& Behavioral Research. Thousand Oaks: Sage Publications.

Valencia, C. (2016). Conciencia situacional y prevención de riesgos delictivos. Recuperado de www.cj-worldnews.com/spain/index.php/en/criminologia30/seguridad/item/2925-conciencia-situacional-y-prevencion-de-riesgosdelictivos 
Revista Iberoamericana de las Ciencias Sociales y Humanísticas

ISSN: 2395 - 7972

\begin{tabular}{|c|c|}
\hline Rol de Contribución & Autor (es) \\
\hline Conceptualización & $\begin{array}{l}\text { Galo Emanuel López Gamboa (principal) } \\
\text { Silvia Alejandra Baeza Aldana (igual) }\end{array}$ \\
\hline Metodología & $\begin{array}{l}\text { Galo Emanuel López Gamboa (principal) } \\
\text { Silvia Alejandra Baeza Aldana (igual) }\end{array}$ \\
\hline Software & N/A \\
\hline Validación & $\begin{array}{l}\text { Galo Emanuel López Gamboa (principal) } \\
\text { Silvia Alejandra Baeza Aldana (igual) }\end{array}$ \\
\hline Análisis Formal & $\begin{array}{l}\text { Galo Emanuel López Gamboa (principal) } \\
\text { Silvia Alejandra Baeza Aldana (igual) }\end{array}$ \\
\hline Investigación & Galo Emanuel López Gamboa \\
\hline Recursos & Silvia Alejandra Baeza Aldana \\
\hline Curación de datos & $\begin{array}{l}\text { Silvia Alejandra Baeza Aldana (principal) } \\
\text { Galo Emanuel López Gamboa (igual) }\end{array}$ \\
\hline $\begin{array}{l}\text { Escritura - Preparación del } \\
\text { borrador original }\end{array}$ & $\begin{array}{l}\text { Galo Emanuel López Gamboa (principal) } \\
\text { Silvia Alejandra Baeza Aldana (igual) }\end{array}$ \\
\hline $\begin{array}{l}\text { Escritura - Revisión y } \\
\text { edición }\end{array}$ & $\begin{array}{l}\text { Silvia Alejandra Baeza Aldana (principal) } \\
\text { Galo Emanuel López Gamboa (igual) }\end{array}$ \\
\hline Visualización & $\begin{array}{l}\text { Galo Emanuel López Gamboa (principal) } \\
\text { Silvia Alejandra Baeza Aldana (igual) }\end{array}$ \\
\hline Supervisión & $\begin{array}{l}\text { Silvia Alejandra Baeza Aldana (principal) } \\
\text { Galo Emanuel López Gamboa (igual) }\end{array}$ \\
\hline Administración de Proyectos & Galo Emanuel López Gamboa \\
\hline Adquisición de fondos & Silvia Alejandra Baeza Aldana \\
\hline
\end{tabular}

